(2) Open Access Full Text Article

REVIEW

\title{
Anxiety and Depression in Patients with Primary Biliary Cholangitis: Current Insights and Impact on Quality of Life
}

\section{Tarika Sivakumar \\ Kris V Kowdley}

Liver Institute Northwest Seattle, Seattle, WA, USA
Correspondence: Kris V Kowdley

Liver Institute Northwest, 3216 NE 45th

PI, Suite 212, Seattle, WA, 98105,

Tel + I 206-536-3030

Fax + I 206-524-0749

Email kkowdley@liverinstitutenw.org

\begin{abstract}
Primary biliary cholangitis (PBC), formerly known as primary biliary cirrhosis, is a chronic cholestatic immune-mediated liver disease characterized by injury to intrahepatic bile ducts that may ultimately progress to cirrhosis and liver failure and result in the need for liver transplant or death without treatment. Ursodeoxycholic acid (UDCA) and obeticholic acid (OCA) are approved therapies for $\mathrm{PBC}$ and are associated with a reduced risk of progression of disease, although patients may continue to experience significant symptoms of pruritus and fatigue independent of liver disease. The two most commonly reported symptoms among patients with $\mathrm{PBC}$ are fatigue and pruritus which may be debilitating, and negatively impact physical, mental, emotional, and social wellbeing. Intense symptom burden has been associated with depressive symptoms, cognitive defects, poor sleep schedules, and social isolation. This literature review explores the presence of anxiety and depressive symptoms in chronic liver disease, the impact of symptom burden on patients' wellbeing, and available pharmaceutical and natural therapies.
\end{abstract}

Keywords: primary biliary cholangitis, depression, anxiety, fatigue, quality of life, chronic liver disease

\section{Clinical Features of PBC Etiology and Pathogenesis}

The cause of PBC is unknown but there are suggested genetic and environmental components. Currently, over $90 \%$ of PBC patients are women, and a majority are Caucasian over the age of 50. ${ }^{1-7}$ An increased prevalence has been reported in monozygotic twins and first-degree relatives of patients with PBC., ${ }^{3,8}$ Geographic clustering of $\mathrm{PBC}$ cases indicates potential causative environmental factors, such as toxins or bacteria. ${ }^{8}$ While the incidence of PBC in northern Europe ranges from 200 to 251 cases per million people, the disease is rare in sub-Saharan Africa and India. ${ }^{9}$

Currently, PBC is diagnosed based on a cholestatic pattern of liver test abnormalities and presence of disease-specific serum antimitochondrial antibodies (AMA); ${ }^{3,10} 95 \%$ of PBC patients test positive for such AMAs and therefore liver biopsy is required for diagnosis only in a small minority of patients. ${ }^{3,10}$ Patients with PBC develop elevated levels of serum bilirubin with advanced disease, which reflects loss of intrahepatic bile ducts over time. ${ }^{1,10}$ Elevated serum alkaline phosphatase (ALP) and/or bilirubin, despite an adequate trial of UDCA, predict an increased risk of liver-related complications and liver-related death or need for 
liver transplant over time. ${ }^{1,11}$ These have been used as surrogate markers for adverse disease outcomes in clinical trials and identify patients who may benefit from OCA as second-line treatment for PBC..$^{1,10}$

Many patients remain asymptomatic for years delaying their diagnosis. ${ }^{1,3,10}$ Many of these undiagnosed patients present with hypercholesteremia, mild pruritus, and Sicca Syndrome. $^{3}$ About $60 \%$ of patients today are diagnosed during this asymptomatic period due to increased availability of diagnostic tools, such as serological testing. ${ }^{12}$ Following diagnosis and treatment with UDCA, many patients can lead a normal life; however, many may continue to experience significant symptom burden independent to liver disease. ${ }^{6}$ Patients who are UDCA intolerant may require other therapies, including investigational drugs.

PBC patients may experience complications such as bone disease, fat soluble vitamin deficiency, hyperlipidemia, fatigue, and pruritus. ${ }^{3}$ About $84 \%$ of PBC patients often exhibit features of a non-hepatic autoimmune disease and $40 \%$ report two or more non-hepatic diseases. ${ }^{6}$ A study showed that $10 \%$ of PBC patients had rheumatoid arthritis, $12 \%$ had Raynaud's syndrome, and 10\% had Sjogren's syndrome. ${ }^{8}$ Between $20 \%$ and $45 \%$ of PBC patients experience osteopenia or osteoporosis which increases the likelihood of fractures and falls, increasing the morbidity of PBC. ${ }^{13}$

Symptoms of PBC can be debilitating, even in the early stages of the disease, and impact health-related quality of life. ${ }^{11}$ As we describe in this review, symptom burden, mental health, age at diagnosis, and lack of response to treatment are all closely interrelated. The aim of this review is to explore the prevalence of anxiety and depression in populations with $\mathrm{PBC}$ and chronic liver disease (CLD), understand the affect these common symptoms have on health-related quality of life, evaluate the impact of liver transplant on PBC patients' physical and mental wellbeing, and explore the current therapies clinicians use when treating $\mathrm{PBC}$ patients.

\section{Natural History of PBC}

The incidence and prevalence of $\mathrm{PBC}$ have increased in recent years to due to widely available diagnostic tools, such as serological testing and liver function tests. ${ }^{3,6}$ While histopathological analysis of the liver is no longer required for diagnosis, it has prognostic value in assessing the stage of the disease and best course of treatment. ${ }^{6} \mathrm{PBC}$ has a variety of clinical presentations, the three most common being a lack of any symptoms, a less aggressive "silent" form in which symptoms may later emerge, and the presence of symptoms. ${ }^{6,11}$ While survival is lower among all of these PBC cohorts when compared to age-matched and gender-matched control groups, some studies have shown that patients who are asymptomatic have better outcomes than those who are symptomatic. ${ }^{6,11}$ Patients who present with PBC at a younger age also usually have worse outcomes and more intense symptom burden. ${ }^{11}$ Development of esophageal varices and hepatocellular carcinoma can also impact the trajectory of $\mathrm{PBC}$ and survival. ${ }^{11}$ A variety of models that assess biochemistries, clinical presentation, and histology have been developed to predict survival. ${ }^{11}$

\section{Symptom Description - Related to QOL}

Many patients report that the most common symptoms of $\mathrm{PBC}$, fatigue and pruritus, can be debilitating and interfere with their ability to complete daily functions. ${ }^{14-20}$ This results in many patients self-reporting depressive symptoms, anxiety, and an overall lower health-related quality of life. ${ }^{14-20}$ Symptomatic patients and patients who are diagnosed earlier in life usually experience greater psychological, social, and physical symptom burden which further negatively impacts their perceived quality of life. ${ }^{14-20}$ Mental health disorders are more common in patients with chronic disease which can negatively impact the trajectory of the disease. Therefore, it is an important aspect of health for clinicians to evaluate when treating PBC patients. $^{21}$

\section{Methods for Quantifying Symptoms and QOL}

Symptom burden and quality of life in patients with PBC are primarily monitored through self-assessments and interviews. Surveys quantify health-related quality of life, anxiety, depressive symptoms, sleep, fatigue, and pruritus in efforts to better understand the disease. Currently, the most widely used survey specific to PBC is the PBC-40, a collection of 40 questions that address various physical, social, and psychological factors that impact patients' health-related quality of life. ${ }^{22}$ Other surveys are included in Table 1. 
Table I Methods for Quantifying Symptom Burden and Health-Related Quality of Life

\begin{tabular}{|c|c|c|c|c|}
\hline \multicolumn{2}{|l|}{ Name } & \multirow{2}{*}{$\begin{array}{l}\text { Type } \\
\text { Self- } \\
\text { assessment }\end{array}$} & \multirow{2}{*}{$\begin{array}{l}\text { Measures } \\
\begin{array}{l}\text { Depressive } \\
\text { symptoms }\end{array}\end{array}$} & \multirow{2}{*}{$\begin{array}{l}\text { Description } \\
\text { Administered to outpatients in a hospital setting to evaluate if they have } \\
\text { anxiety or depression and the severity of the disorder }\end{array}$} \\
\hline $\begin{array}{l}\text { Hospital Anxiety and } \\
\text { Depression Scale }\end{array}$ & HADS & & & \\
\hline Beck Depression Inventory & $\mathrm{BDI}$ & $\begin{array}{l}\text { Self- } \\
\text { assessment }\end{array}$ & $\begin{array}{l}\text { Depressive } \\
\text { symptoms }\end{array}$ & $\begin{array}{l}21 \text { question self-assessment widely used in clinical and research settings } \\
\text { to evaluate and scale depressive symptoms outlined by the Diagnostic } \\
\text { and Statistical Manual for Mental Disorders }{ }^{67}\end{array}$ \\
\hline $\begin{array}{l}\text { Diagnostic and Statistical } \\
\text { Manual for Mental } \\
\text { Disorders }\end{array}$ & DSM IV & $\begin{array}{l}\text { Diagnostic } \\
\text { tool }\end{array}$ & $\begin{array}{l}\text { Various } \\
\text { mental } \\
\text { disorders }\end{array}$ & $\begin{array}{l}\text { Diagnostic criteria defined by the American Psychiatric Association for } \\
\text { evaluating psychiatric disorders }\end{array}$ \\
\hline $\begin{array}{l}\text { Penn State Worry } \\
\text { Questionnaire }\end{array}$ & PSWQ & $\begin{array}{l}\text { Self- } \\
\text { assessment }\end{array}$ & $\begin{array}{l}\text { General } \\
\text { anxiety } \\
\text { disorder }\end{array}$ & $\begin{array}{l}\text { Consistent and reliable } 16 \text { item questionnaire used to evaluate } \\
\text { Generalized Anxiety Disorder (GAD) }\end{array}$ \\
\hline Visual Analogue Scale & VAS & $\begin{array}{l}\text { Self- } \\
\text { assessment }\end{array}$ & Pain & $\begin{array}{l}10 \mathrm{~cm} \text { self-assessment scale used to measure disease-related pain, } \\
\text { ranging from no pain to severe pain }{ }^{70}\end{array}$ \\
\hline Fisk Fatigue Severity Scale & FFSS & $\begin{array}{l}\text { Self- } \\
\text { assessment }\end{array}$ & Fatigue & $\begin{array}{l}\text { Self-reported measurement of disease-related physical, social, and } \\
\text { cognitive fatigue }{ }^{17}\end{array}$ \\
\hline $\begin{array}{l}\text { Wurzburg depletion } \\
\text { Inventory for multiple } \\
\text { sclerosis }\end{array}$ & WEIMUS & $\begin{array}{l}\text { Self- } \\
\text { assessment }\end{array}$ & Fatigue & $\begin{array}{l}\text { Fatigue scale developed for German patients with multiple sclerosis } \\
\text { used to evaluate PBC patients where PBC specific scales were } \\
\text { unavailable }{ }^{18}\end{array}$ \\
\hline Symptom Checklist-90 & SCL-90 & $\begin{array}{l}\text { Self- } \\
\text { assessment }\end{array}$ & $\begin{array}{l}\text { Various } \\
\text { symptoms }\end{array}$ & $\begin{array}{l}\text { Questionnaire used to evaluate } 90 \text { distress symptoms across nine } \\
\text { psychological domains } s^{71}\end{array}$ \\
\hline Epworth Sleepiness Scale & ESS & $\begin{array}{l}\text { Self- } \\
\text { assessment }\end{array}$ & $\begin{array}{l}\text { Excessive } \\
\text { daytime } \\
\text { sleepiness }\end{array}$ & $\begin{array}{l}\text { Eight question self-assessment used to evaluate excessive daytime } \\
\text { sleepiness (EDS) }\end{array}$ \\
\hline $\begin{array}{l}\text { Hamilton Rating Scale for } \\
\text { Depression }\end{array}$ & HRSD & $\begin{array}{l}\text { Self- } \\
\text { assessment }\end{array}$ & $\begin{array}{l}\text { Depressive } \\
\text { Symptoms }\end{array}$ & 17 question survey used to evaluate severity of depression ${ }^{72}$ \\
\hline $\begin{array}{l}\text { Center for Epidemiologic } \\
\text { Studies Depression Rating } \\
\text { Scale }\end{array}$ & CES-D & $\begin{array}{l}\text { Self- } \\
\text { assessment }\end{array}$ & $\begin{array}{l}\text { Depressive } \\
\text { symptoms }\end{array}$ & $\begin{array}{l}\text { A 4-factor-20-question self-assessment used to measure depressive } \\
\text { symptoms, similar to } \mathrm{BDI}^{73}\end{array}$ \\
\hline $\begin{array}{l}\text { Short Form-36 Health } \\
\text { Survey }\end{array}$ & SF-36 & $\begin{array}{l}\text { Self- } \\
\text { assessment }\end{array}$ & $\begin{array}{l}\text { Health- } \\
\text { related } \\
\text { quality of life }\end{array}$ & 36 item questionnaire used to evaluate health-related quality of life ${ }^{74}$ \\
\hline
\end{tabular}

\section{Depression and Anxiety in Chronic Liver Disease (CLD) Patients}

Depression and anxiety are common amongst patients experiencing chronic liver disease (CLD). ${ }^{23,24}$ Patients experiencing chronic illness are several times more likely to experience depression than the normal population, including symptoms such as emotional suffering, loss of purpose, and impaired ability to function and complete daily activities. ${ }^{23,25}$ Patients with CLDs are at risk for complications, comorbidities, and developing cirrhosis and hepatocellular carcinomas (HCC), which may result in the need for a liver transplant or death. $^{23,26}$ The depressive symptoms and mental disorders that manifest through extrahepatic pathways in CLD patients greatly impact the trajectory of the chronic illness and the patients' ability to cope with the disease, which may result in a lower perceived quality of life. ${ }^{25-27}$

\section{Fatigue and Pruritus \\ Fatigue}

Fatigue is the most reported symptom that PBC patients experience, and can range from mild to debilitating. ${ }^{14,15,28}$ The fatigue is often independent of disease severity, 
meaning that even patients with early-stage PBC may experience intense fatigue. ${ }^{14,15,28}$ Fatigue can have detrimental effects on patients' mental, physical, and social health by preventing individuals from participating in daily activities and interacting with others. ${ }^{13}$ The negative impact of fatigue on every aspect of life largely contributes to patients reporting low self-perceived health-related quality of life. ${ }^{13}$

A study that assessed 327 PBC patients over the course of 7.2 years reported that $44 \%$ of the patients experienced moderate to severe symptoms with $25 \%$ experiencing mild to severe fatigue, as measured using the PBC-40 survey. ${ }^{16}$ Increased fatigue was positively correlated with body mass index, pruritus, cirrhosis, and increased use of prescribed medications, including antipruritic drugs and antidepressants. ${ }^{16}$ The stage of the disease at diagnosis also impacted patient reported fatigue, with patients diagnosed at a later stage experiencing a greater symptom burden, possibly due to lack of management. ${ }^{16}$

A different study of 116 PBC patients reported that 99 patients experienced fatigue, and $50 \%$ of those patients reported fatigue as being one of the worst, if not the worst, symptom of their disease. ${ }^{17}$ Patients' self-reported fatigue and symptom burden, as measured through the Fatigue Impact Scale (FIS) and Symptom Checklist-90 (SCL-90) assessments, noted that PBC patients scores were significantly higher than controls, indicating a large symptom burden. ${ }^{17}$ Of the 116 total patients, 52 scored above 10 on the Beck Depression Inventory (BDI) scale, indicating depressive symptoms. ${ }^{17}$ While self-reported depressive symptoms are not equivalent to a clinician's diagnosis of depression, this study suggests that the presence of fatigue is highly correlated with depressive symptoms in PBC patients. ${ }^{17}$ When clinicians approach therapies for mitigating fatigue, they must also provide treatments that target depressive symptoms.

A study monitoring fatigue and quality of life through the FIS and Short Form-36 (SF-36) survey in 81 female $\mathrm{PBC}$ patients found that fatigue was a primary predictor of quality of life due to the impact it has on physical, social, emotional, and psychological factors in a patient's life. ${ }^{15}$

The UK-PBC cohort assessed symptoms in 2002 PBC patients using the Hospital Anxiety and Depression Scale (HADS), Epworth Sleepiness Scale (ESS), and Orthostatic Grading Scale. ${ }^{29}$ Of the 1203 patients who reported significant fatigue, $55 \%$ also reported significant cognitive symptoms, suggesting that there may be a relationship between fatigue and cognitive defects. ${ }^{29}$
Many studies have found that fatigue in PBC is associated with depressive symptoms, poor cognitive health, and poor health-related quality of life. ${ }^{14-17,28}$ Overall, it is commonly reported that fatigue negatively impacts patients' social life and mental health due to social isolation and inability to complete day-to-day tasks. ${ }^{14-17,28}$ Fatigue may prevent patients from completing physical activity, interacting with friends and family, maintaining a normal sleep schedule, having a job, and completing normal tasks in general. ${ }^{14-17,28}$

\section{Pruritus}

Pruritus, or itch, is a common debilitating symptom in patients with chronic liver disease. As many as $60 \%$ to $70 \%$ of patients with $\mathrm{PBC}$ report experiencing pruritus. ${ }^{20}$ Pruritus can occur at any stage of $\mathrm{PBC}$ and is independent of disease severity. ${ }^{20,30}$ Although pruritus is described as mild by most patients, a significant proportion report severe pruritus that interferes with maintaining a sleep schedule and completing daily functions. ${ }^{30}$ Pruritus is associated with fatigue, depression, and may even be associated with suicidal ideation, in severe cases. ${ }^{30}$

Most patients with chronic liver disease (60-80\%) also experience sleep disorders such as insomnia, excessive daytime sleepiness (EDS), or difficulty staying asleep. $^{31,32}$ According to a study with 48 female PBC patients and 48 female control subjects monitored using the Pittsburgh Sleep Quality Index (PSQI) and ESS, PBC patients spend 30 more minutes in bed at night than controls and experience significantly more sleep latency. ${ }^{31,33}$ PBC patients also perceive that they sleep for a significantly shorter amount of time than controls. ${ }^{31}$ These sleep problems may be caused by a disruption of the melatonin metabolism pathway that the liver partially regulates or by decreased hepatic blood flow and competition with bilirubin in the intrahepatic transport system. ${ }^{31,32}$ PBC patients often report that pruritus affects their ability to sleep and therefore pruritus often coincides with EDS and sleep disturbances. ${ }^{20}$

\section{Depression and Anxiety in PBC Patients - Possible Mechanisms}

Many patients with $\mathrm{PBC}$ experience a lower self-assessed health-related quality of life due to significant symptom burden which impacts mental health. Depression and anxiety in PBC may have a multifactorial basis. Current hypotheses have implicated disrupted neurotransmitter 
synthesis, changes in brain structure seen on imaging, or directly the result from the symptom burden of fatigue and pruritus. ${ }^{18,34-36}$

\section{Neurotransmitter Synthesis}

A 2001 study evaluated serum amino acid concentrations of 45 PBC patients in an Erasmus Medical Center outpatient hepatology clinic. ${ }^{17}$ This study included 73 healthy individuals and 22 patients with untreated hepatitis $C$ viral infections as the control groups. ${ }^{17}$ This study sought to evaluate if neurotransmitter synthesis is impaired in patients with cholestasis by measuring serum tyrosine, tryptophan, and branched amino acid levels. ${ }^{17}$ These amino acid levels were further evaluated in relation to fatigue and quality of life on the FFSS, VAS, and SF-36 self-assessment scales. ${ }^{17}$ The severity of fatigue, the most prominent, debilitating symptom of $\mathrm{PBC}$, is independent of the severity of the disease, suggesting that extrahepatic factors may be involved. ${ }^{17}$ Plasma tyrosine concentrations were inversely correlated with fatigue. ${ }^{17}$ Tryptophan is the amino acid precursor to serotonin which stabilizes mood and helps regulate appetite and sleep. ${ }^{37}$ Plasma tryptophan levels were also significantly negatively correlated with the cognitive domain of fatigue, as measured on the FFSS scale. ${ }^{17} \mathrm{PBC}$ patients with tyrosine levels higher than controls had lower fatigue scores; it is interesting to note that patients with cirrhosis also had significantly high levels of plasma tyrosine. ${ }^{17}$ Overall, lower levels of tyrosine and tryptophan were significantly associated with higher levels of fatigue and worse quality of life. These symptoms are often associated with mental health disorders in patients with chronic disease such as PBC. ${ }^{17,38}$ Although this study did not directly assess depressive symptoms and anxiety in PBC patients, there is an understood link between neurotransmitter levels and depression and anxiety. ${ }^{17,39-41}$

A separate study that evaluated the role of psychological factors in PBC-associated fatigue reported that the group of "high-fatigue" patients were significantly more likely to report depressive and anxious symptoms. ${ }^{21}$

\section{Brain Structure}

The symptom burden and mental disorders often associated with PBC may result from physical changes in brain structure. ${ }^{18,42} \mathrm{~A}$ study of 20 female noncirrhotic PBC patients and 21 age-matched controls evaluated the relationship between the symptom burden patients experience and changes in their brain's deep gray matter using resting state functional magnetic resonance imaging (rsfMRI) and resting state functional connectivity (rsFC). ${ }^{17}$ The specific structures that were evaluated were the amygdala, hippocampus, thalamus and putamen, structures imperative in learning, memory, and processing emotions. ${ }^{17}$ PBC patients exhibited reduced activity of the putamen in the basal ganglia on functional magnetic resonance imaging (fMRI). ${ }^{17}$ Other fMRI studies have also linked disruptions in the amygdala, hippocampus, basal ganglia and thalamus of the limbic system with depression and altered connectivity in deep gray matter of the brain in patients with $\mathrm{PBC}$ when compared to controls. ${ }^{17,43,44}$ Another study of 14 pre-cirrhotic patients and controls found a relationship between differences in the magnetization transfer ratios of the globus pallidus, a structure below the cortex near the putamen, and fatigue. ${ }^{40}$ Manganese normally excreted through the bile ducts has been implicated as a cause of fatigue in PBC. ${ }^{40,45}$

\section{Self-Assessment Vs Clinical Diagnosis of Mental Disorders in PBC Patients}

Self-assessments are administered to PBC patients to quantify symptom severity to help clinicians better understand patient symptom burden. Many surveys are developed from the Diagnostic and Statistical Manual for Mental Disorders (DSM IV) which defines the criteria for diagnosing mental disorders. ${ }^{18,35}$ However, answers to these questionnaires are subjective. ${ }^{18,35}$

One study conducted on 20 PBC patients and 20 agematched control subjects in a German population reported that PBC patients are severely more affected by depressive symptoms and psychological symptoms, as measured by the BDI and SCL-90 scales, respectively. ${ }^{17}$ Further analysis of the SCL-90 showed that PBC patients engage in or experience significantly more somatization, compulsive behavior, depressive, anxiety, aggressiveness, and other psychiatric symptoms. ${ }^{17}$

A different study of 92 Dutch patients with either PBC or primary sclerosing cholangitis (PSC), another cholestatic liver disease, wanted to assess whether the prevalence of depression was between $20 \%$ and $45 \%$, as other studies reported. ${ }^{34}$ This study used multiple separate tools to measure depressive symptom severity and confirm diagnosis. Of the 55 PBC patients screened, 21 patients scored above 10 on the BDI scale, exceeding the cut-off for screening for depressive disorder. ${ }^{34}$ However, of those 21 
patients, only 2 met the standard for diagnosis as outlined by the DSM-IV. ${ }^{34}$ The results of this study suggest that although the BDI, Hamilton Rating Scale for Depression (HRSD), and Center for Epidemiologic Studies (CES-D) questionnaires were developed to evaluate depression, each is effective in measuring depressive symptom severity, whereas the DSM-IV is used to clinically diagnose depressive disorder. ${ }^{34}$ Although PBC patients may experience increased depressive symptom burden, it does not equate to a clinical diagnosis with depression. ${ }^{34}$ This study therefore concluded that the prevalence of depressive disorder is similar in $\mathrm{PBC}$ populations compared to the general population. ${ }^{34}$ The manifestation of depressive symptoms may result from PBC symptom burden or be unrelated to $\mathrm{PBC}$ altogether. ${ }^{18,35}$

\section{PBC and Liver Transplant - Early Vs End Stage}

Liver transplantation is associated with excellent long-term survival with a 5-year and 10-year survival rate of $80 \%$ and $70 \%$, respectively. ${ }^{12,46} \mathrm{PBC}$ may recur post-transplant but does not diminish long-term outcomes overall. ${ }^{47}$ Recurrent $\mathrm{PBC}$ is usually diagnosed via liver biopsy of the allograft. ${ }^{12}$ In a study with 785 PBC patients who underwent liver transplant across North America and Europe, PBC recurred in $22 \%$ and $36 \%$ of patients 5 -years and 10 -years postoperation, respectively. ${ }^{48}$ Patients who are younger at the time of $\mathrm{PBC}$ diagnosis or liver transplant are more likely to experience $\mathrm{PBC}$ recurrence post-transplant. ${ }^{46,48}$

The most common post-operative complications within the first-year post-transplant are bone pain and fractures, hypertension, and renal failure. ${ }^{12}$ Two years post-transplant, $76 \%$ of patients were able to perform daily activities and selfassessed their health as good or very good. ${ }^{12}$ Over time, liver transplant can ease PBC symptoms and improve quality of life. In a study with $25 \mathrm{PBC}$ patients on the transplant waitlist and 75 patients post-transplant, physical quality of life improved significantly after 5 years post-transplant. ${ }^{47}$ However, mental quality of life remained below the national average, as assessed through a multivariate analysis of fatigue, depression, coping, and education. ${ }^{47}$

\section{Anxiety and Depression - Management and Recommendations}

\section{Pharmaceutical Management}

PBC patients are commonly prescribed antidepressants, anti-anxiety medications, and anti-pruritic drugs to help manage their mental health and symptom burden. ${ }^{49}$ These prescribed medications include mirtazapine, hydroxyzine, and sertraline. ${ }^{49}$

A 2018 analysis of a UK PBC cohort of 1177 patients identified between April 1974 and May 2007 showed that $7.3 \%$ of patients were diagnosed with depression prior to their diagnosis with $\mathrm{PBC}$ and $6.7 \%$ were diagnosed with depression after their diagnosis with $\mathrm{PBC} ;{ }^{49} 11.1 \%$ of patients took antidepressants before their diagnosis with PBC and $24.6 \%$ after. ${ }^{49}$ PBC patients with depression had an increased risk of mortality compared to those without depression. ${ }^{49}$ After adjusting for UDCA use, gender, severity of depression, and alcohol intake, treatment with the antidepressant mirtazapine was correlated with a decreased risk of decompensated cirrhosis, need for liver transplant, and death. ${ }^{49}$ Mirtazapine is an atypical antidepressant with effects on both central and peripheral networks, and acts as an antagonist on norepinephrine, serotonin, and histamine receptors. ${ }^{50}$

A separate study evaluated the effects of mirtazapine on autoimmune hepatitis, a chronic liver disease that has been noted to overlap with PBC. ${ }^{50}$ This study dosed mice with concanavalin A (Con A) to imitate human $\mathrm{AIH} .{ }^{50,51}$ Mirtazapine reduced liver damage and significantly increased hepatic innate immune responses in Con A-induced mice. ${ }^{50}$ The overall pharmacology of mirtazapine in improving hepatic outcomes requires further exploration.

Hydroxyzine is an antihistamine used to alleviate pruritus and anxiety in patients with liver disease. ${ }^{52}$ However, side effects include fatigue, drowsiness, and dry mouth. ${ }^{52}$ The side effects of hydroxyzine can ultimately worsen symptom burden, including depression. ${ }^{52}$ In a study including $8 \mathrm{PBC}$ patients treated with hydroxyzine at different doses over the course of 24 hours, two of the patients reported blurred vision, dizziness, and dry mouth. ${ }^{52}$ The results of this study indicated that patients with $\mathrm{PBC}$ take longer to eliminate hydroxyzine, which may lead to more negative side effects. Hydroxyzine has also rarely been associated with torsade de pointes and increased cardiovascular risk. ${ }^{53}$ In alleviating pruritus in PBC patients, hydroxyzine should not be used at the first line of treatment.

Sertraline is a selective serotonin reuptake inhibitor (SSRI) used to treat anxiety and depression. ${ }^{54}$ In patients with $\mathrm{PBC}$, sertraline may reduce pruritus and improve overall well-being. ${ }^{54}$ An initial dose of $50-100 \mathrm{mg}$ of sertraline is typically used for this indication. ${ }^{54}$ A study 
followed 40 PBC patients over an average of 7.5 years. $^{54}$ Of the 40 patients, 7 experienced pruritus and were prescribed sertraline; 6 of 7 patients reported significantly less or no pruritus after taking a stable dose of sertraline for over 6 months. $^{54}$ These patients also decreased their dose or completely stopped taking any other anti-pruritic medications as well, suggesting that sertraline may be effective in lessening cholestatic pruritus. ${ }^{54}$ Another study treated 21 patients with chronic pruritus suffering from $\mathrm{PBC}$, PSC, viral hepatitis $\mathrm{C}$ (HCV), or post-necrotic cirrhosis with 75-100mg of sertraline daily and assessed pruritus using the Visual Analogue Scale (VAS). ${ }^{55}$ Sertraline was well tolerated and resulted in reduced pruritus compared to increased pruritus "scores" among placebo-treated patients. ${ }^{55}$

In addition to sertraline, other SSRIs such as citalopram, escitalopram, fluoxetine, and paroxetine are often taken by patients with chronic liver disease to manage anxiety and depression. ${ }^{56}$ SSRIs are the most commonly prescribed group of antidepressants to patients with CLDs. ${ }^{56}$ Selective noradrenergic reuptake inhibitors (SNRIs) including duloxetine and venlafaxine are also prescribed to CLD patients for anxiety and depression. ${ }^{56}$ While SSRIs and SNRIs may improve anxiety and depressive symptoms, more research in relation to PBC symptoms, such as fatigue, pruritus, and liver health, must be conducted. $^{56}$

Bezafibrate, a drug used to target hypercholesterolemia and hypertriglyceridemia, has also been used to treat $\mathrm{PBC}$ in combination with UDCA. ${ }^{57,58}$ A study of 369 PBC patients showed that UDCA and bezafibrate combination therapy was effective in decreasing liver enzymes in patients non-responsive to UDCA only. ${ }^{58}$ Another study was conducted on $22 \mathrm{PBC}$ patients over a 6-month period. Patients taking $600 \mathrm{mg} /$ day of UDCA and $400 \mathrm{mg} /$ day of bezafibrate had significantly lower ALP levels at the end of treatment. ${ }^{59}$ In addition, pruritus disappeared in one of seven patients receiving bezafibrate, suggesting that bezafibrate may improve health-related quality of life in $\mathrm{PBC}$ patients who do not respond to UDCA. ${ }^{59}$

Nalfurafine hydrochloride, an opioid receptor antagonist, has been studied in patients with chronic liver and kidney disease who experience pruritus. ${ }^{60,61}$ In a study, 44 PBC patients were administered 2.5 micrograms of nalfurafine daily. After a 12-week period, patients' PBC-40 and VAS scores assessing for pruritus significantly decreased, indicating that nalfurafine may be effective in targeting pruritus. $^{61}$
Although both drugs may be effective in improving physical and mental health outcomes in PBC patients, bezafibrate and nalfurafine hydrochloride must be studied more extensively in relation to PBC treatment, anxiety, and depression in patients with chronic liver disease.

S-Adenosyl-L-Methionine (SAMe) has also been tried as a therapeutic option in PBC patients. ${ }^{62} 24$ PBC patients were simultaneously treated with $13-15 \mathrm{mg} / \mathrm{kg}$ of UDCA and $1200 \mathrm{mg}$ of SAMe for 6 months. By the end of treatment, ALP, gamma glutamyl transferase (GGT), and cholesterol levels had significantly improved. ${ }^{62}$ Patients' fatigue and pruritus scores also significantly improved, as measured by the PBC-40 questionnaire. ${ }^{62}$ In addition, anxiety and depression scores, as measured by HADS, trended towards improving but changes were not statistically significant. ${ }^{62}$ This study suggests that concomitant therapy of UDCA and SAMe may benefit PBC patients' health-related quality of life, anxiety, and depression. ${ }^{62}$ However, further research is required.

Currently, there are no approved medications that target fatigue and there is no evidence that UDCA or OCA lessen fatigue.

\section{Natural Therapies and Recommendations}

In addition to utilizing available pharmaceutical interventions in managing depression, anxiety, and pruritus, $\mathrm{PBC}$ patients have the option of engaging in natural therapies to alleviate symptom burden. Natural therapies include exercise, yoga, meditation, maintaining a healthy diet, and not smoking. ${ }^{63}$ Such natural therapies have general health benefits as well, such as reducing the risk of heart disease and stroke. $^{63}$

Chronically ill patients, including those with PBC, report difficulty engaging in aerobic exercise due to physical limitations and symptom burden, such as pruritus and fatigue. ${ }^{63}$ Exercise is recommended for physical and mental health, as endorphins released during physical activity can improve mood and perception of quality of life. ${ }^{63}$

Meditation, breathing exercises, and yoga have been suggested to improve anxiety and depressive symptoms in PBC. ${ }^{64,65}$

\section{Conclusion}

Primary biliary cholangitis is a rare chronic liver disease characterized by inflammation of the small intrahepatic bile ducts. Late diagnosis, lack of management, or intolerance to the current available pharmaceutical therapies may result in cirrhosis and liver failure. It is imperative for 
PBC patients to be closely monitored by clinicians with an understanding of the disease.

A majority of PBC patients experience the two most common and debilitating symptoms of the disease: fatigue and pruritus. Intense symptom burden may impact patients' ability to complete daily activities and maintain social connections. Symptom burden has such a large effect on mental, social, emotional, and physical health that patients report self-isolation, cognitive changes, inability to sleep, and anxiety and depressive symptoms. Many patients report depression and anxiety on hospital administered self-assessments but do not meet the standard for formal diagnosis, as outlined in the DSM IV. Despite not having a formal diagnosis with depression, many patients are prescribed anti-depressants. It is unclear as to whether the antidepressants improve other symptoms such as fatigue and pruritus.

Overall, PBC patients self-report a lower health-related quality of life when compared to control age-matched, gender-matched populations. Therefore, when approaching possible treatments for disease management, it is very important for clinicians to consider the effects of $\mathrm{PBC}$ beyond liver damage. While there are certain pharmaceuticals that clinicians recommend in managing depression and pruritus, patients may engage in natural therapies, such as routine exercise and meditation, to improve their symptom burden and overall cardiorespiratory health. However, there is still a need for more specific symptomdirected therapies that are widely effective to alleviate fatigue and pruritus. By targeting these symptoms, clinicians may improve patients' anxiety, depressive symptoms, and overall health-related quality of life.

\section{Abbreviations}

PBC, Primary biliary cholangitis; UDCA, Ursodeoxycholic acid; OCA, Obeticholic acid; AMA, Antimitochondrial antibody; ALP, Alkaline phosphatase; CLD, Chronic liver disease; HCC, Hepatocellular carcinoma; HADS, Hospital Anxiety and Depression Scale; BDI, Beck Depression Inventory; DSM IV, Diagnostic and Statistical Manual for Mental Disorders; PSWQ, Penn State Worry Questionnaire; VAS, Visual Analogue Scale; FFSS, Fisk Fatigue Severity Scale; WEIMUS, Wurzburg depletion Inventory for multiple sclerosis; SCL-90, Symptom Checklist-90; ESS, Epworth Sleepiness Scale; EDS, Excessive daytime sleepiness; HRSD, Hamilton Rating Scale for Depression; CES-D, Center for Epidemiologic Studies; SF-36, Short Form-36
Health Survey; UK-PBC, United Kingdom primary biliary cholangitis cohort; rsfMRI, Resting state functional magnetic resonance imaging; rsFC, Resting state functional connectivity; fMRI, Functional magnetic resonance imaging; Con A, Concanavalin A; PSC, Primary sclerosing cholangitis; $\mathrm{HCV}$, Chronic viral hepatitis $\mathrm{C}$; GGT, Gamma glutamyl transferase; SSRI, Selective serotonin reuptake inhibitor; SNRI, Selective noradrenergic reuptake inhibitor.

\section{Disclosure}

Dr Kris V Kowdley reports grants, personal fees from CymaBay, grants, personal fees from Genfit, grants, personal fees from Gilead, grants from GSK, grants, personal fees from HighTide, grants, personal fees from Intercept, outside the submitted work. The authors report no other conflicts of interest.

\section{References}

1. Pandit SSH. Primary Biliary Cholangitis. Treasure Island (FL): StatPearls Publishing; 2021.

2. Lu M, Zhou Y, Haller IV, et al. Increasing Prevalence of Primary Biliary Cholangitis and Reduced Mortality With Treatment. Clin Gastroenterol Hepatol. 2018;16(8):1342-1350.e1341. doi:10.1016/j. cgh.2017.12.033

3. Reshetnyak VI. Primary biliary cirrhosis: clinical and laboratory criteria for its diagnosis. World $J$ Gastroenterol. 2015;21 (25):7683-7708. doi:10.3748/wjg.v21.i25.7683

4. Hohenester S, Oude-Elferink RPJ, Beuers U. Primary biliary cirrhosis. Semin Immunopathol. 2009;31(3):283-307.

5. Poordad F. Diagnosis, Treatment, and Monitoring of Patients With Primary Biliary Cholangitis. Gastroenterol Hepatol (N Y). 2016;12 (9):561-564.

6. Al-Harthy N, Kumagi T. Natural history and management of primary biliary cirrhosis. Hepatic Med. 2012;4:61-71.

7. Hirschfield GM. Diagnosis of primary biliary cirrhosis. Best Pract Res Clin Gastroenterol. 2011;25(6):701-712. doi:10.1016/j. bpg.2011.10.005

8. Gershwin ME, Selmi C, Worman HJ, et al. Risk factors and comorbidities in primary biliary cirrhosis: a controlled interview-based study of 1032 patients. Hepatology. 2005;42(5):1194-1202. doi:10.1002/hep.20907

9. Podda M, Selmi C, Lleo A, Moroni L, Invernizzi P. The limitations and hidden gems of the epidemiology of primary biliary cirrhosis. J Autoimmun. 2013;46:81-87. doi:10.1016/j.jaut.2013.06.015

10. Aguilar MTCD, Chascsa DM. Update on Emerging Treatment Options for Primary Biliary Cholangitis. Hepat Med. 2020; Volume 12(12):69-77. doi:10.2147/HMER.S205431

11. Parés A, Rodés J. Natural history of primary biliary cirrhosis. Clin Liver Dis. 2003;7(4):779-794. doi:10.1016/S1089-3261(03)00100-4

12. Navasa M, Forns X, Sánchez V, et al. Quality of life, major medical complications and hospital service utilization in patients with primary biliary cirrhosis after liver transplantation. J Hepatol. 1996;25 (2):129-134. doi:10.1016/S0168-8278(96)80064-X

13. Trivedi HD, Danford CJ, Goyes D, Bonder A. Osteoporosis in Primary Biliary Cholangitis: prevalence, Impact and Management Challenges. Clin Exp Gastroenterol. 2020;13:17-24. doi:10.2147/ CEG.S204638 
14. Griffiths L, Jones DE. Pathogenesis of primary biliary cirrhosis and its fatigue. Dig Dis. 2014;32(5):615-625. doi:10.1159/000360515

15. Sogolow ED, Lasker JN, Short LM. Fatigue as a major predictor of quality of life in women with autoimmune liver disease: the case of primary biliary cirrhosis. Womens Health Issues. 2008;18 (4):336-342. doi:10.1016/j.whi.2007.12.005

16. Al-Harthy N, Kumagi T, Coltescu C, Hirschfield GM. The specificity of fatigue in primary biliary cirrhosis: evaluation of a large clinic practice. Hepatology. 2010;52(2):562-570. doi:10.1002/hep.23683

17. Huet PM, Deslauriers J, Tran A, Faucher C, Charbonneau J. Impact of fatigue on the quality of life of patients with primary biliary cirrhosis. Am J Gastroenterol. 2000;95(3):760-767. doi:10.1111/ j.1572-0241.2000.01857.x

18. Zenouzi R, von der Gablentz J, Heldmann M, et al. Patients with primary biliary cholangitis and fatigue present with depressive symptoms and selected cognitive deficits, but with normal attention performance and brain structure. PLoS One. 2018;13(1):e190005e0190005. doi:10.1371/journal.pone.0190005

19. Bergasa NV. Pruritus and fatigue in primary biliary cirrhosis. Clin Liver Dis. 2003;7(4):879-900. doi:10.1016/S1089-3261(03)00105-3

20. Trivedi HD, Lizaola B, Tapper EB, Bonder A. Management of Pruritus in Primary Biliary Cholangitis: a Narrative Review. Am $J \quad$ Med. 2017;130(6):744.e741-744.e747. doi:10.1016/j.amjm ed.2017.01.037

21. Blackburn P, Freeston M, Baker CR, Jones DE, Newton JL. The role of psychological factors in the fatigue of primary biliary cirrhosis. Liver Int. 2007;27(5):654-661. doi:10.1111/j.1478-3231.2007.015 $00 . \mathrm{x}$

22. Jacoby A, Rannard A, Buck D, et al. Development, validation, and evaluation of the PBC-40, a disease specific health related quality of life measure for primary biliary cirrhosis. Gut. 2005;54 (11):1622-1629. doi:10.1136/gut.2005.065862

23. Thombs BD, Taillefer SS, Hudson M, Baron M. Depression in patients with systemic sclerosis: a systematic review of the evidence. Arthritis Rheum. 2007;57(6):1089-1097. doi:10.1002/ art. 22910

24. Evans DL, Charney DS, Lewis L, et al. Mood disorders in the medically ill: scientific review and recommendations. Biol Psychiatry. 2005;58(3):175-189.

25. Ziegelstein RC, Fauerbach JA, Stevens SS, Romanelli J, Richter DP, Bush DE. Patients with depression are less likely to follow recommendations to reduce cardiac risk during recovery from a myocardial infarction. Arch Intern Med. 2000;160(12):1818-1823. doi:10.1001/ archinte.160.12.1818

26. Huang X, Liu X, Yu Y. Depression and Chronic Liver Diseases: are There Shared Underlying Mechanisms? Front Mol Neurosci. 2017;10:134. doi:10.3389/fnmol.2017.00134

27. Benrud-Larson LM, Haythornthwaite JA, Heinberg LJ, et al. The impact of pain and symptoms of depression in scleroderma. Pain. 2002;95(3):267-275. doi:10.1016/S0304-3959(01)00409-2

28. Jopson L, Jones DE. Fatigue in Primary Biliary Cirrhosis: prevalence, Pathogenesis and Management. Dig Dis. 2015;33(Suppl 2):109-114. doi:10.1159/000440757

29. Phaw NA, Dyson JK, Mells G, Jones D. Understanding Fatigue in Primary Biliary Cholangitis. Dig Dis Sci. 2021;66(7):2380-2386. doi:10.1007/s10620-020-06502-0

30. Tajiri K, Shimizu Y. Recent advances in the management of pruritus in chronic liver diseases. World $j$ Gastroenterol. 2017;23 (19):3418-3426. doi:10.3748/wjg.v23.i19.3418

31. Newton JL, Gibson GJ, Tomlinson M, Wilton K, Jones D. Fatigue in primary biliary cirrhosis is associated with excessive daytime somnolence. Hepatology. 2006;44(1):91-98. doi:10.1002/hep.21230

32. Shah NM, Malhotra AM, Kaltsakas G. Sleep disorder in patients with chronic liver disease: a narrative review. J Thorac Dis. 2020;12 (Suppl 2):S248-S260. doi:10.21037/jtd-cus-2020-012
33. Boyes J, Drakatos P, Jarrold I, Smith J, Steier J. The use of an online Epworth Sleepiness Scale to assess excessive daytime sleepiness. Sleep Breath. 2017;21(2):333-340. doi:10.1007/s11325-016-1417-x

34. Ter Borg PC, Fekkes D, Vrolijk JM, van Buuren HR. The relation between plasma tyrosine concentration and fatigue in primary biliary cirrhosis and primary sclerosing cholangitis. BMC Gastroenterol. 2005;5:11. doi:10.1186/1471-230X-5-11

35. van Os E, van den Broek WW, Mulder PG. Depression in patients with primary biliary cirrhosis and primary sclerosing cholangitis. J Hepatol. 2007;46(6):1099-1103. doi:10.1016/j.jhep.2007.01.036

36. Labenz C, Huber Y, Michel M, et al. Nonalcoholic Fatty Liver Disease Increases the Risk of Anxiety and Depression. Hepatol Commun. 2020;4(9):1293-1301. doi:10.1002/hep4.1541

37. Strasser B, Gostner JM, Fuchs D. Mood, food, and cognition: role of tryptophan and serotonin. Curr Opin Clin Nutr Metab Care. 2016;19 (1):55-61. doi:10.1097/MCO.0000000000000237

38. McGuire J, Ross GL, Price H, Mortensen N, Evans J, Castell LM. Biochemical markers for post-operative fatigue after major surgery. Brain Res Bull. 2003;60(1-2):125-130. doi:10.1016/S0361-9230(03) 00021-2

39. Kraus C, Castrén E, Kasper S, Lanzenberger R. Serotonin and neuroplasticity - Links between molecular, functional and structural pathophysiology in depression. Neurosci Biobehav Rev. 2017;77:317-326. doi:10.1016/j.neubiorev.2017.03.007

40. Grace AA. Dysregulation of the dopamine system in the pathophysiology of schizophrenia and depression. Nat Rev Neurosci. 2016;17 (8):524-532. doi:10.1038/nrn.2016.57

41. Hirschfeld RM. History and evolution of the monoamine hypothesis of depression. J Clin Psychiatry. 2000;61(Suppl 6):4-6.

42. Mosher VAL, Swain MG, Pang JXQ, et al. Primary Biliary Cholangitis Alters Functional Connections of the Brain's Deep Gray Matter. Clin Transl Gastroenterol. 2017;8(7):e107-e107. doi:10.1038/ctg.2017.34

43. Julia L. Newton Cognitive impairment in primary biliary cirrhosis: symptom impact and potential etiology. Hepatology. 2008;48 (2):541-549. doi:10.1002/hep.22371

44. Miller AHJJ, Drake DF, Tian H, Unger ER, Pagnoni G. Decreased Basal Ganglia Activation in Subjects with Chronic Fatigue Syndrome: association with Symptoms of Fatigue. PLoS One. 2014;9(5):48.

45. Forton DM, Patel N, Prince M, et al. Fatigue and primary biliary cirrhosis: association of globus pallidus magnetisation transfer ratio measurements with fatigue severity and blood manganese levels. Gut. 2004;53(4):587-592. doi:10.1136/gut.2003.016766

46. Akamatsu N, Sugawara Y. Primary biliary cirrhosis and liver transplantation. Intractable Rare Dis Res. 2012;1(2):66-80.

47. Pells G, Mells GF, Carbone M, et al. The impact of liver transplantation on the phenotype of primary biliary cirrhosis patients in the UK-PBC cohort. $J$ Hepatol. 2013;59(1):67-73. doi:10.1016/j. jhep.2013.02.019

48. Lasker JN, Sogolow ED, Short LM, Sass DA. The impact of biopsychosocial factors on quality of life: women with primary biliary cirrhosis on waiting list and post liver transplantation. $\mathrm{Br} J$ Health Psychol. 2011;16(3):502-527. doi:10.1348/135910710X527964

49. Shaheen AA, Kaplan GG, Almishri W, et al. The impact of depression and antidepressant usage on primary biliary cholangitis clinical outcomes. PLoS One. 2018;13(4):e0194839. doi:10.1371/journal. pone. 0194839

50. Almishri W, Shaheen AA, Sharkey KA, Swain MG. The Antidepressant Mirtazapine Inhibits Hepatic Innate Immune Networks to Attenuate Immune-Mediated Liver Injury in Mice. Front Immunol. 2019;10:803. doi:10.3389/fimmu.2019.00803

51. Wang HX, Liu M, Weng SY, et al. Immune mechanisms of Concanavalin A model of autoimmune hepatitis. World J Gastroenterol. 2012;18(2):119-125. doi:10.3748/wjg.v18.i2.119 
52. Simons FE, Watson WT, Chen XY, Minuk GY, Simons KJ. The pharmacokinetics and pharmacodynamics of hydroxyzine in patients with primary biliary cirrhosis. J Clin Pharmacol. 1989;29 (9):809-815. doi:10.1002/j.1552-4604.1989.tb03424.x

53. Pate J, Gutierrez JA, Frenette CT, et al. Practical strategies for pruritus management in the obeticholic acid-treated patient with PBC: proceedings from the 2018 expert panel. BMJ Open Gastroenterol. 2019;6(1):e00256. doi:10.1136/bmjgast-2018000256

54. Browning J, Combes B, Mayo MJ. Long-term efficacy of sertraline as a treatment for cholestatic pruritus in patients with primary biliary cirrhosis. Am J Gastroenterol. 2003;98(12):2736-2741. doi:10.1111/ j.1572-0241.2003.08662.x

55. Mayo MJ, Handem I, Saldana S, Jacobe H, Getachew Y, Rush AJ. Sertraline as a first-line treatment for cholestatic pruritus. Hepatology. 2007;45(3):666-674. doi:10.1002/hep.21553

56. Mullish BH, Kabir MS, Thursz MR, Dhar A. Review article: depression and the use of antidepressants in patients with chronic liver disease or liver transplantation. Aliment Pharmacol Ther. 2014;40 (8):880-892. doi:10.1111/apt.12925

57. Monk JP, Todd PA. A review of its pharmacodynamic and pharmacokinetic properties, and therapeutic use in hyperlipidaemia. Drugs. 1987;33(6):539-576. doi:10.2165/00003495-198733060-00002

58. Agrawal R, Majeed M, Attar BM, et al. Effectiveness of bezafibrate and ursodeoxycholic acid in patients with primary biliary cholangitis: a meta-analysis of randomized controlled trials. Ann Gastroenterol. 2019;32(5):489-497.

59. Kanda T, Yokosuka O, Imazeki F, Saisho H. Bezafibrate treatment: a new medical approach for PBC patients? J Gastroenterol. 2003;38 (6):573-578. doi:10.1007/s00535-002-1102-7

60. Nakao K, Mochizuki H. Nalfurafine hydrochloride: a new drug for the treatment of uremic pruritus in hemodialysis patients. Drugs Today. 2009;45(5):323-329. doi:10.1358/dot.2009.45.5.1377595

61. Yagi M, Tanaka A, Namisaki T, et al. Is patient-reported outcome improved by nalfurafine hydrochloride in patients with primary biliary cholangitis and refractory pruritus? A post-marketing, single-arm, prospective study. J Gastroenterol. 2018;53(10):1151-1158. doi:10.1007/s00535-018-1465-z

62. Wunsch E, Raszeja-Wyszomirska J, Barbier O, Milkiewicz M, Krawczyk M, Milkiewicz P. Effect of S-adenosyl-L-methionine on liver biochemistry and quality of life in patients with primary biliary cholangitis treated with ursodeoxycholic acid. A prospective, open label pilot study. J Gastrointestin Liver Dis. 2018;27(3):273-279. doi:10.15403/jgld.2014.1121.273.icz
63. Hallsworth K, Jopson L, Jones DE, Trenell MI. Exercise therapy in primary biliary cirrhosis: the importance of moving while sitting on a surgical waiting list-a case study. Frontline Gastroenterol. 2016;7 (3):167-169. doi:10.1136/flgastro-2015-100672

64. Williams H, Simmons LA, Tanabe P. Mindfulness-Based Stress Reduction in Advanced Nursing Practice: a Nonpharmacologic Approach to Health Promotion, Chronic Disease Management, and Symptom Control. J Holist Nurs. 2015;33(3):247-259. doi:10.1177/ 0898010115569349

65. Venkatesh HN, Ravish H, Delphine Silvia CW, Srinivas H. Molecular Signature of the Immune Response to Yoga Therapy in Stress-related Chronic Disease Conditions: an Insight. Int J Yoga. 2020;13(1):9-17. doi:10.4103/ijoy.IJOY_82_18

66. Zigmond AS, Snaith RP. The hospital anxiety and depression scale. Acta Psychiatr Scand. 1983;67(6):361-370. doi:10.1111/j.16000447.1983.tb09716.x

67. García-Batista ZE, Guerra-Peña K, Cano-Vindel A, HerreraMartínez SX, Medrano LA. Validity and reliability of the Beck Depression Inventory (BDI-II) in general and hospital population of Dominican Republic. PLoS One. 2018;13(6):e0199750. doi:10.1371/ journal.pone.0199750

68. Pull CB. DSM-IV guidebook. American Psychiatric Association [DSM-IV]. Encephale. 1995;21(5):15-20.

69. Meyer TJ, Miller ML, Metzger RL, Borkovec TD. Development and validation of the Penn State Worry Questionnaire. Behav Res Ther. 1990;28(6):487-495. doi:10.1016/0005-7967(90)90135-6

70. Haefeli M, Elfering A. Pain assessment. Eur Spine J. 2006;15 Suppl 1(Suppl1):S17-S24. doi:10.1007/s00586-005-1044-x

71. Sereda Y, Dembitskyi S. Validity assessment of the symptom checklist SCL-90-R and shortened versions for the general population in Ukraine. BMC Psychiatry. 2016;16(1):300. doi:10.1186/s12888-0161014-3

72. Zimmerman M, Martinez JH, Young D, Chelminski I, Dalrymple K. Severity classification on the Hamilton Depression Rating Scale. $J$ Affect Disord. 2013;150(2):384-388. doi:10.1016/j.jad.2013.04.028

73. Carleton RN, Thibodeau MA, Teale MJN, et al. The Center for Epidemiologic Studies Depression Scale: a Review with a Theoretical and Empirical Examination of Item Content and Factor Structure. PLoS One. 2013;8(3):e58067. doi:10.1371/journal. pone.0058067

74. Lins L, Carvalho FM. SF-36 total score as a single measure of health-related quality of life: scoping review. SAGE Open Med. 2016;4:2050312116671725. doi:10.1177/2050312116671725
Hepatic Medicine: Evidence and Research

\section{Publish your work in this journal}

Hepatic Medicine: Evidence and Research is an international, peerreviewed, open access journal covering all aspects of adult and pediatric hepatology in the clinic and laboratory including the following topics: Pathology, pathophysiology of hepatic disease; Investigation and treatment of hepatic disease; Pharmacology of drugs used for the treatment of hepatic disease. Issues of patient safety and quality of care will also be considered. The manuscript management system is completely online and includes a very quick and fair peer-review system, which is all easy to use. Visit http://www.dovepress.com/ testimonials.php to read real quotes from published authors. 